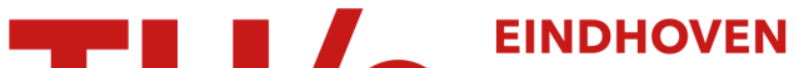

\section{Spontaneous angular momentum generation of two- dimensional fluid flow in an elliptic geometry}

\section{Citation for published version (APA):}

Keetels, G. H., Clercx, H. J. H., \& Heijst, van, G. J. F. (2008). Spontaneous angular momentum generation of two-dimensional fluid flow in an elliptic geometry. Physical Review E - Statistical, Nonlinear, and Soft Matter Physics, 78(3), 036301-1/7. [036301]. https://doi.org/10.1103/PhysRevE.78.036301

DOI:

10.1103/PhysRevE.78.036301

Document status and date:

Published: 01/01/2008

\section{Document Version:}

Publisher's PDF, also known as Version of Record (includes final page, issue and volume numbers)

\section{Please check the document version of this publication:}

- A submitted manuscript is the version of the article upon submission and before peer-review. There can be important differences between the submitted version and the official published version of record. People interested in the research are advised to contact the author for the final version of the publication, or visit the $\mathrm{DOI}$ to the publisher's website.

- The final author version and the galley proof are versions of the publication after peer review.

- The final published version features the final layout of the paper including the volume, issue and page numbers.

Link to publication

\section{General rights}

Copyright and moral rights for the publications made accessible in the public portal are retained by the authors and/or other copyright owners and it is a condition of accessing publications that users recognise and abide by the legal requirements associated with these rights.

- Users may download and print one copy of any publication from the public portal for the purpose of private study or research.

- You may not further distribute the material or use it for any profit-making activity or commercial gain

- You may freely distribute the URL identifying the publication in the public portal.

If the publication is distributed under the terms of Article 25fa of the Dutch Copyright Act, indicated by the "Taverne" license above, please follow below link for the End User Agreement:

www.tue.nl/taverne

Take down policy

If you believe that this document breaches copyright please contact us at:

openaccess@tue.nl

providing details and we will investigate your claim. 


\title{
Spontaneous angular momentum generation of two-dimensional fluid flow in an elliptic geometry
}

\author{
G. H. Keetels, ${ }^{1, *}$ H. J. H. Clercx, ${ }^{1,2}$ and G. J. F. van Heijst ${ }^{1}$ \\ ${ }^{1}$ Department of Physics, Eindhoven University of Technology, P.O. Box 513, 5600 MB Eindhoven, The Netherlands \\ ${ }^{2}$ Department Applied Mathematics, University of Twente, P.O. Box 217, 7500 AE Enschede, The Netherlands
}

(Received 26 October 2007; revised manuscript received 14 June 2008; published 3 September 2008)

\begin{abstract}
Spontaneous spin-up, i.e., the significant increase of the total angular momentum of a flow that initially has no net angular momentum, is very characteristic for decaying two-dimensional turbulence in square domains bounded by rigid no-slip walls. In contrast, spontaneous spin-up is virtually absent for such flows in a circular domain with a no-slip boundary. In order to acquire an understanding of this strikingly different behavior observed on the square and the circle, we consider a set of elliptic geometries with a gradual increase of the eccentricity. It is shown that a variation of the eccentricity can be used as a control parameter to tune the relative contribution of the pressure and viscous stresses in the angular momentum balance. Direct numerical simulations demonstrate that the magnitude of the torque can be related to the relative contribution of the pressure. As a consequence, the number of spin-up events in an ensemble of slightly different initial conditions depends strongly on the eccentricity. For small eccentricities, strong and rapid spin-up events are observed occasionally, whereas the majority of the runs do not show significant spin-up. Small differences in the initial condition can result in a completely different evolution of the flow and an appearance of the end state of the decay process. For sufficiently large eccentricities, all the runs in the ensemble demonstrate strong and rapid spin-up, which is consistent with the flow development on the square. It is verified that the number of spin-up events for a given eccentricity does not depend on the Reynolds number of the flow. This observation is consistent with the conjecture that it is the pressure on the domain boundaries that drives the spin-up processes.
\end{abstract}

DOI: 10.1103/PhysRevE.78.036301

PACS number(s): 47.20.Ky, 47.11.Kb, 47.27.De, 47.32.C-

\section{INTRODUCTION}

Many characteristic phenomena of two-dimensional (2D) turbulence, such as the formation of coherent structures, vorticity filamentation, and the turbulent dual cascades, are usually analyzed theoretically or studied numerically in the absence of rigid boundaries. Moreover, in experimental studies it is often assumed that rigid walls are located at a sufficient distance from the measurement area so that any influence of the walls is assumed negligible. Interaction of $2 \mathrm{D}$ flows with a no-slip boundary can, however, dramatically affect the evolution of both decaying and forced 2D turbulence; for a recent overview, see [1]. In the present paper, we focus on the spontaneous production of angular momentum $L$ (the precise definition will follow), and associated spontaneous symmetry breaking of the flow, due to the interaction of decaying $2 \mathrm{D}$ turbulence with elliptical, no-slip sidewalls.

One of the first remarkable results in this field hinting at the special role of the angular momentum was obtained numerically by Li et al. $[2,3]$. They reported that the decay scenario of 2D turbulence in a circular geometry with a noslip boundary depends strongly on the net angular momentum contained by the initial flow field. Any angular momentum production due to flow-wall interactions seems insignificant for this particular geometry. It was found that the late-time state depends strongly on the amount of angular momentum introduced during the initialization of the flow. In particular, an initial flow field without significant angular momentum yields after a rapid self-organization process a quadrupolar structure filling the circular container. This

\footnotetext{
*h.j.h.clercx@tue.nl.
}

structure eventually evolves toward a dipolar structure in the late-time flow evolution. On the other hand, an initial turbulent velocity field that contains some net angular momentum evolves into a large monopolar structure that eventually fills the entire container. The numerical predictions of $\mathrm{Li}$ et al. $[3,4]$ have been confirmed experimentally by Maassen $e t$ al. [5]. Typical initial integral-scale Reynolds numbers in the numerical and experimental studies were $\operatorname{Re}=U W / \nu \approx 2$ $\times 10^{3}$, with $U$ the rms velocity, $W$ the size of the container, and $\nu$ the kinematic viscosity of the fluid.

Similar simulations and experiments have been conducted for decaying $2 \mathrm{D}$ turbulence in square domains by Clercx et al. $[6,7]$. These studies gave an essentially different picture. It was observed that the no-slip boundaries of the square container exert a net torque on the fluid such that the flow, which has initially no significant amount of angular momentum, acquires angular momentum during the decay process. As a consequence, rapid production of angular momentum can result in a large monopolar or tripolar vortex completely filling the domain later on in the flow evolution, a clear sign of spontaneous symmetry breaking of the flow. It was reported that in an ensemble of simulations with an initial Reynolds number of $\operatorname{Re}=2 \times 10^{3}$, a part of the runs showed strong spin-up effects [6]. However, an ensemble of runs with initially $\operatorname{Re}=10^{4}$ revealed that all the simulations show a flow evolution following the scenario with sudden and strong spin-up [7], although the flow in the container after it has spun up consists basically of a sea of smaller-scale vortices on top of a domain-filling swirling flow. Approximately one-half of the runs showed the emergence of a clockwise swirl, and the other half an anticlockwise motion; thus on average, symmetry breaking is absent, as is to be expected. 


\section{ANGULAR MOMENTUM PRODUCTION}

In a recent study of the circular case [8], it was shown that the production of angular momentum on a circle is negligible for higher Reynolds numbers, up to $\mathrm{Re}=5 \times 10^{4}$, as well. Montgomery [9] suggests that the elliptical geometry in particular would be a good starting point to further investigate the behavior of bounded 2D fluids. A hint to explain the dramatic influence of the shape of the no-slip boundary may be found in the angular momentum balance,

$$
\frac{d L}{d t}=\frac{1}{\rho} \oint_{\partial \mathcal{D}} p(\boldsymbol{r}, t) \boldsymbol{r} \cdot d s+\frac{1}{\operatorname{Re}} \oint_{\partial \mathcal{D}} \omega(\boldsymbol{r}, t)(\boldsymbol{r} \cdot \boldsymbol{n}) d s,
$$

where the angular momentum is defined as

$$
L=\int_{\mathcal{D}} \boldsymbol{r} \times \boldsymbol{u} d A=\int_{\mathcal{D}}(x v-y u) d A .
$$

Here, we introduced a Cartesian coordinate system $(x, y)$ with origin in the center of the container, and $\boldsymbol{u}=(u, v)$ with respect to this coordinate system. Furthermore, $p$ represents the pressure, $\rho$ is the fluid density, and $\omega=\frac{\partial v}{\partial x}-\frac{\partial u}{\partial y}$ is the vorticity associated with the 2D velocity field $\boldsymbol{u}$. The first term on the right-hand side of Eq. (1) represents the pressure contribution and the second term the effect of viscous shear and normal stresses. An important result can directly be conjectured from Eq. (1): the pressure term is essentially zero on the boundary of the circular domain, since $\boldsymbol{r} \cdot d \boldsymbol{s} \equiv \mathbf{0}$. This signifies that although the pressure distribution over the domain boundary may be asymmetric, it does not yield a net torque to the interior fluid. For flows on the square domain, however, it is reported by Clercx et al. [7] that it is essentially the pressure term that guides the spin-up process as it is orders of magnitude larger than the viscous stress contribution represented by the second term on the right-hand side in Eq. (1).

To investigate the generalization toward an elliptic geometry, it is useful to represent Eq. (1) in the cylindrical elliptic coordinate system $(\eta, \xi)$, where a constant $\xi$ and variable $\eta$ in the range $0<\eta<2 \pi$ describe an elliptic curve. For convenience, we keep the major axis $a \equiv 1$ while the minor halfaxis $b=1-\delta$ is in the range $0<b \leqslant 1$ in the following. The angular momentum balance then reads

$$
\frac{d L}{d t}=\frac{\delta(\delta-2)}{2 \rho} \oint_{\partial D} p(\eta) \sin (2 \eta) d \eta+\frac{(1-\delta)}{\operatorname{Re}} \oint_{\partial D} \omega(\eta) d \eta .
$$

The pressure term in Eq. (3) is of order $\delta$ and will grow linearly with the deviation from the circle geometry. The contribution of the viscous stresses remains small. Note that in an elliptic geometry, a broken symmetry in the pressure distribution over the domain boundary can, in principle, yield a global spin-up of the fluid in the interior. Summarizing: by making a small change $\delta$ from a circular to an elliptic geometry, we can tune the relative contribution of the pressure term and the viscous stresses in the angular momentum balance, and eventually determine whether the pressure contribution is actually responsible for the spontaneous spin-up.
Moreover, it allows us to determine a critical value for the minor half-axis $b_{\text {crit }}$ below which always spin-up occurs.

For normalization of the angular momentum, it is important to know the maximum amount of angular momentum that can be present on an elliptic domain for a given amount of the total kinetic energy $E=\frac{1}{2} \int_{D}\left(u^{2}+v^{2}\right) d A$. For arbitrary geometries (and irrespective of the boundary conditions) it is helpful to introduce a limit to the angular momentum with a Schwarz inequality, yielding

$$
L \leqslant\|\boldsymbol{r}\|_{2}\|\boldsymbol{u}\|_{2}=\|\boldsymbol{r}\|_{2} \sqrt{2 E},
$$

where $\|\cdot\|_{2}$ denotes the $L_{2}$ norm. The right-hand side of inequality (4) equals the amount of angular momentum if the fluid is in solid body rotation. Impermeability of the elliptic boundary prevents fluid from obtaining a full solid body state. It is, however, possible to derive an upper bound for the angular momentum that is consistent with the impermeability of the elliptic boundary. By virtue of the incompressibility condition $\boldsymbol{\nabla} \cdot \boldsymbol{u}=\mathbf{0}$ we can introduce a stream function according to $u=\partial_{y} \psi$ and $v=-\partial_{x} \psi$. An impermeable boundary can be modeled by setting $\psi=0$ at the boundary. The angular momentum and total kinetic energy can then be formulated as $L=2 \int_{\mathcal{D}} \psi d A$ and $E=\frac{1}{2} \int_{\mathcal{D}} \omega \psi d A$. Using standard variational techniques [10], it is straightforward to show that the variational problem $L[\psi]$ with constraint $E[\psi]=1$ yields a Poisson equation, $\nabla^{2} \psi=-2 \Omega$ with $\psi=0$ on the boundary. The Lagrange multiplier $\Omega$ equals the angular velocity in the case of solid body rotation on the circle. The solution normalized with the amount of angular momentum of the same fluid in solid body rotation reads

$$
\widetilde{L}=\frac{2}{b / a+a / b}
$$

where $a$ and $b$ represent the major and minor half-axes of the ellipse, respectively. Figure 1 shows the solutions of the Poisson problem on the ellipse and the corresponding upper bound for the angular momentum. The streamline pattern has the appearance of a single cell filling the entire container.

Keeping in mind the tendency to axisymmetrization of a domain filling vortex, we also consider an alternative streamline pattern (Fig. 1, bottom). It is computed by conformal mapping (modified Joukowski) of the solid body rotation on a circle to an elliptic geometry. A single concentric cell appears in the center, while the streamlines become eccentric when moving radially outward.

Both patterns show that for small deviations of the minor half-axis from the unit circle $(b>0.7)$, the amount of angular momentum that can be reached is more than $90 \%$ of solid body rotation. For larger eccentricities, i.e., $\delta \gtrsim 0.3$, on the other hand, the angular momentum vanishes much faster. Recall that the upper bound is derived by only demanding impermeability of the boundary, allowing a free-slip velocity at the boundary. Incorporation of a no-slip boundary condition in the variational problem is not possible. Demanding an extra condition, i.e., zero circulation, $\Gamma=\int_{\mathcal{D}} \omega d A=0$, yields the same Poisson equation though with Cauchy boundary conditions, which are too restrictive on a closed surface [10]. 


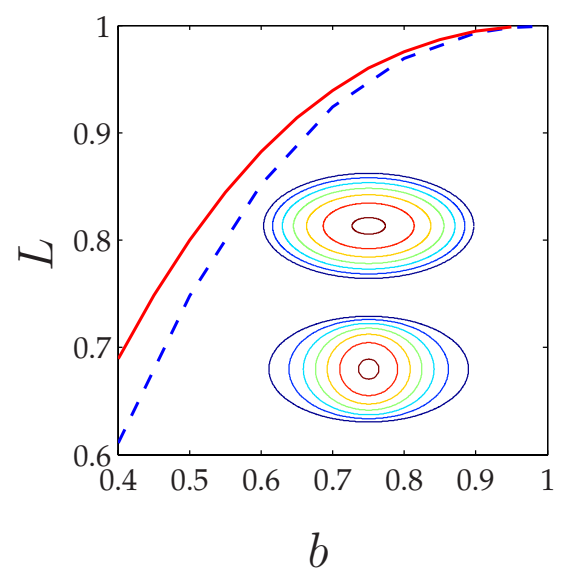

FIG. 1. (Color online) Upper bound (solid line) for the angular momentum in an elliptic geometry, normalized with angular momentum of the fluid in solid body rotation, versus size of the minor half-axis $b$ while the major half-axis $a=1$ is fixed. The inset shows the streamlines for $b=0.5$ for the Poisson solution (top) and the streamlines obtained by conformal mapping of a uniform rotation on the circle to an ellipse (bottom). The dashed line denotes angular momentum of the conformal mapping result.

\section{NUMERICAL METHOD}

The numerical results are obtained by a Fourier spectral solver combined with an immersed boundary technique, known as "volume-penalization" or "Brinkman penalization" to incorporate the no-slip boundary condition. The concept proposed by Arquis and Caltagirone [11] is to model a solid obstacle with no-slip boundaries as a porous obstacle with an extremely small permeability. The flow domain $\Omega_{f}$ is embedded in a computational domain $\Omega$, such that $\Omega_{f}=\Omega \backslash \overline{\Omega_{s}}$, where $\Omega_{s}$ represents the volume of porous objects. The interaction with the porous objects is modeled by adding a Darcy drag term to the incompressible Navier-Stokes equations (unit density $\rho=1$ ) locally inside $\Omega_{s}$. This gives the penalized Navier-Stokes equation defined for $\boldsymbol{x} \in \Omega$,

$$
\partial_{t} \mathbf{u}+(\mathbf{u} \cdot \nabla) \mathbf{u}+\nabla p-\nu \Delta \mathbf{u}+\frac{1}{\epsilon} H \mathbf{u}=0,
$$

which is accompanied by the continuity equation for $\boldsymbol{x} \in \Omega$,

$$
\boldsymbol{\nabla} \cdot \boldsymbol{u}=0
$$

where $\epsilon$ is the penalization parameter and $H$ represents a mask function defined as

$$
H= \begin{cases}1 & \text { if } \mathbf{x} \in \bar{\Omega}_{s} \\ 0 & \text { if } \mathbf{x} \in \Omega_{f} .\end{cases}
$$

Note that inside the obstacle $\Omega_{s}$, Darcy drag is added and inside the flow domain $\Omega_{f}$, the usual Navier-Stokes equation is considered. The initial velocity is defined inside the flow domain $\Omega_{f}$. Inside the obstacle, the initial condition can be extended to the porous obstacle by setting the initial velocity equal to zero inside the domain $\Omega_{s}$. As a result, the initial condition is properly defined on the entire computational domain $\Omega$. In this study, the mask function is chosen such that the elliptic flow domain is sufficiently embedded inside the computational domain $\Omega$. By simply changing the shape of the mask function, it is possible to solve another elliptic geometry with a different eccentricity. This is employed by fixing the major half-axis $a=1$ and varying the minor halfaxis $b$. In contrast to other immersed boundary techniques, the method is fully theoretically justified $[12,13]$. The penalization error is proportional to $\sqrt{\epsilon}$. Various numerical benchmark studies are available [13-15] and some 2D turbulence studies have already adopted the method $[8,16]$. The Fourierspectral scheme with volume penalization applied in the present study is validated by a detailed convergence analysis on vortex-wall collisions [17]. It was found that it is possible to make the penalization error smaller than the truncation error. Note that the penalization parameter is actually an arbitrary parameter. If an appropriate time scheme is applied, unusual restrictions on the time-step are avoided as well.

Important features of the method are the straightforward implementation of (curved) no-slip boundaries and the efficient computation of 2D turbulence on arbitrary closed domains with parallellized Fourier pseudospectral codes. The flow is initialized by the same procedure as used in the study of Clercx et al. [7] on spontaneous angular momentum production in a square geometry. The initial condition consists of 100 nearly equal-sized Gaussian vortices with a radius 0.05 normalized with the major half-axis $a$ of the ellipses $(a=1)$ and vortex amplitude $\omega_{\max } \simeq 100$ normalized with rms velocity $U=1$ and the major half-axis. Half of the vortices have positive circulation and the other vortices have negative circulation. The vortices are placed on a regular lattice, well away from the boundaries. The symmetry is slightly broken by slight displacement of the vortex centers. In Fourier transform space, certain coefficients are set equal to zero such that the initial angular momentum is zero within machine precision. A smoothing function [7] is applied to ensure that the initial flow is consistent with the no-slip boundary condition.

The ensemble simulations are conducted with a total number of $1024^{2}$ Fourier modes. The penalization parameter is $\epsilon=10^{-8}$ and the time step is $\delta t=10^{-4}$. The following values for minor half-axis $b$ have been considered: $b=1.0,0.95,0.9$, 0.8 , and 0.7 . For each case, 12 ensemble runs are performed. The flow is initialized such that the rms velocity $U=1$. The majority of the runs has an initial Reynolds number of Re $=a U / \nu=10^{4}$. For one case, $b=0.9$, the Reynolds number is increased to $\mathrm{Re}=2 \times 10^{4}$. These runs require a larger resolution up to $2048^{2}$ Fourier modes. To check if convergence is achieved, lower spatial resolution computations have been performed for the same initial condition for each value of the minor half-axis $b$. Furthermore, it is verified that the enstrophy dissipation length scale, defined as $2 \pi\left(\nu^{3} / \chi\right)^{1 / 6}$ with $\chi$ denoting the enstrophy dissipation rate per unit area, is well resolved. The required resolutions are consistent with the convergence study of the numerical scheme based on vortexwall interaction $[17,18]$.

\section{DECAYING 2D TURBULENCE IN ELLIPTIC GEOMETRIES}

Snapshots of the vorticity field from a decaying turbulence simulation in an elliptic domain with minor half-axis 

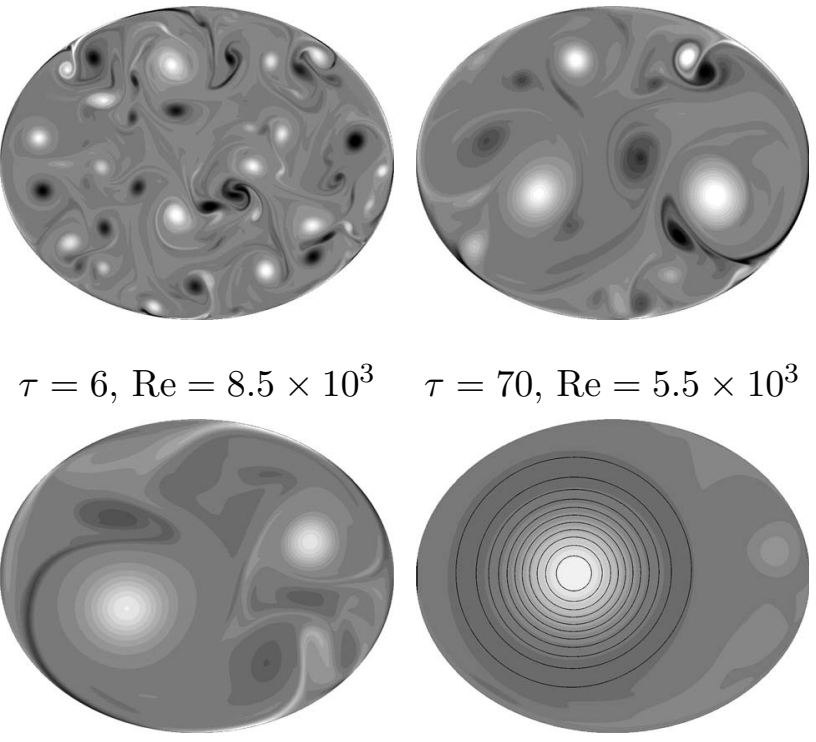

$\tau=200, \operatorname{Re}=3.3 \times 10^{3} \tau=500, \operatorname{Re}=2.1 \times 10^{3}$

FIG. 2. Vorticity plots of a run with an initial Reynolds number $\operatorname{Re}=10^{4}$ and minor half-axis $b=0.8$. White indicates positive vorticity, black negative. For $\tau=500$ also the contour lines of the stream function $\psi$ are shown with an increment of 0.02 . Time is made dimensionless by the turnover time of the initial vortices. Spatial resolution is $1024^{2}$.

$b=0.8$ are displayed in Fig. 2. The initial integral-scale Reynolds number is $\operatorname{Re}=10^{4}$, which eventually decreased to $\mathrm{Re}$ $\approx 2 \times 10^{3}$ at the end of the simulation. The first stage of the decay process is characterized by intense vortex-wall interactions and vortex merger events in the interior of the flow domain. Later the vortex density decreases, while a largescale monopolar vortex starts to fill the interior of the elliptic domain. In Fig. 3, the Reynolds number $\operatorname{Re}=a U / \nu$ $=a \sqrt{2 E(t) / a b \pi} / \nu$ and integral length scale $\mathcal{L}(t)=\sqrt{E(t) / Z(t)}$ [where $Z(t)=1 / 2 \int_{\mathcal{D}} \omega^{2} d A$ denotes the total entropy] are given. The Reynolds number is larger than 2000 for $\tau$

\section{Reynolds number Length scale}
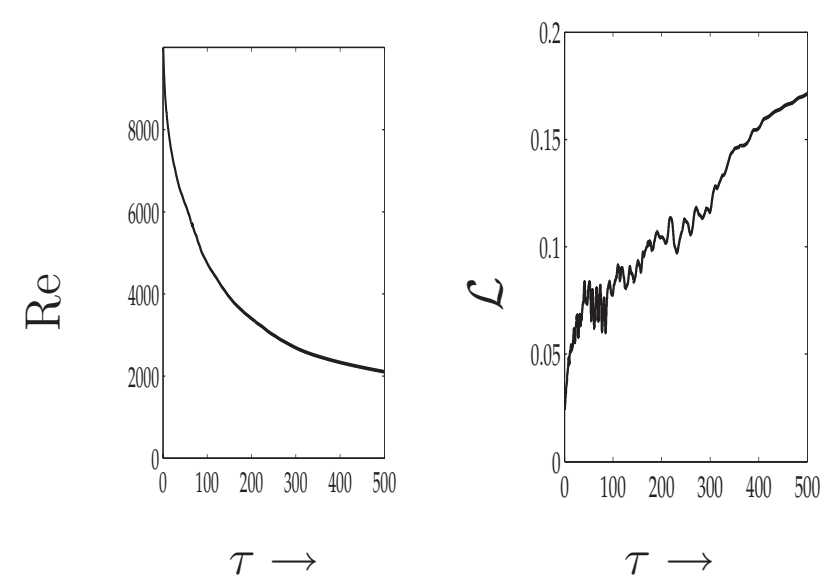

FIG. 3. Reynolds number Re and the integral length scale $\mathcal{L}$ of the simulation shown in Fig. 2.

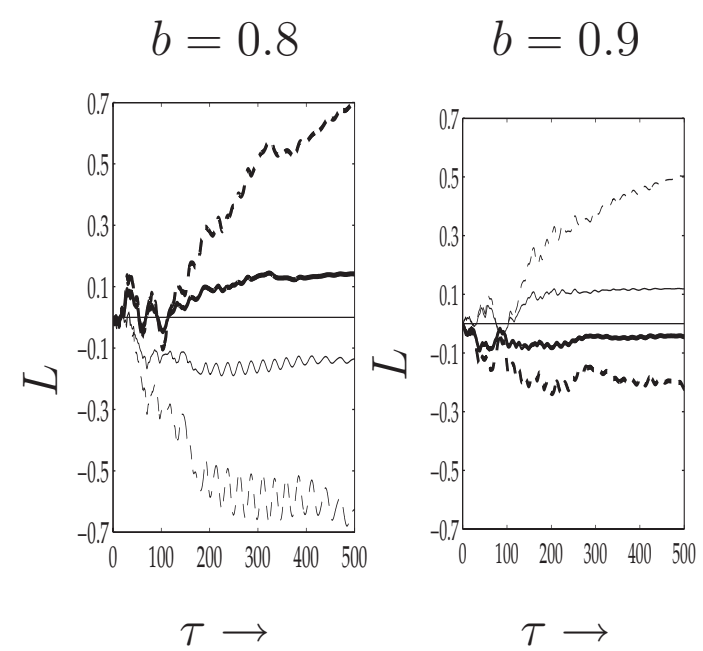

FIG. 4. Angular momentum in an elliptic geometry versus time. Angular momentum is normalized with uniform rotation using $L_{s b}$ at $\tau=0$ (solid line) and $L_{s b}(t)$ (dashed line). Thick lines in the lefthand panel correspond with the run shown in Fig. 2.

$<500$, where $\tau$ is defined as the turnover time of the initial vortices. This implies that the large-scale vortex develops in the nonlinear regime. (Note that for $\tau \rightarrow \infty$, the flow is finally governed by viscous dynamics, which selects the slowest dissipating mode or fundamental Stokes mode.) The integral length scale grows very rapidly in the beginning of the simulation due to merger events and dissipation of entropy in the interior. The growth rate of the integral length scale is suppressed between $80<\tau<300$ due to production of entropy at the domain boundaries. Finally, the integral length scale increases rapidly, which can be associated with the relaxation to the large-scale vortex in Fig. 2 at $\tau=500$.

Note that the emergence of a large monopolar vortex in the interior of the flow domain, as shown in Fig. 2, inevitably implies a net angular momentum. The angular momentum can be normalized by using $L_{s b}=\|\boldsymbol{r}\|_{2}\|\boldsymbol{u}\|_{2}$ with $\|\boldsymbol{r}\|_{2}$ $=1 / 2 \sqrt{b\left(1+b^{2}\right) \pi}$ and $\|\boldsymbol{u}\|_{2}=\sqrt{2 E}$ either determined by the initial energy $E_{0}$ or the instantaneous energy $E(t)$. The first normalization does not take into account viscous dissipation, and although the normalized angular momentum will be virtually constant after spin-up, it eventually decays to zero as the flow dissipates. On the other hand, the second normalization procedure compensates for viscous decay, and in the long-time limit $\left(\tau \gg 10^{3}\right)$ the normalized angular momentum will always approach a certain finite value due to viscous relaxation. Figure 4 shows the normalized angular momentum versus time for two sizes of the minor half-axis. Note that the left-hand panel in Fig. 4 shows the corresponding curves (with thick lines) for the run presented in Fig. 2. It can be observed in Fig. 4 that the angular momentum normalized with $L_{s b}=\|\boldsymbol{r}\|_{2} \sqrt{2 E_{0}}$ (solid line) is produced early and becomes more or less constant after $\tau \gtrsim 300$. Note that in this paper, the quasistationary end state of the nonlinear regime is considered. In the limit of $\tau \rightarrow \infty$, the angular momentum will eventually vanish due to viscous decay. The corresponding curve for the angular momentum of the run shown in Fig. 2 normalized with $L_{s b}(t)=\|\boldsymbol{r}\|_{2} \sqrt{2 E(t)}$ (dashed line) reveals that at $\tau=500$, more than $70 \%$ of uniform-like rotation has been 
TABLE I. Ensemble averaged amplitude $\langle A\rangle$, where $A$ is defined as the maximum for $\tau<500$ of the normalized angular momentum $L_{s b}(t)$. Conditional average $\langle A\rangle_{c}$ over the runs in the ensemble that show strong spin-up of the flow. Standard deviation $\sigma$ of $\dot{L}$ for different sizes of the minor half-axis $b$. The error in $\langle A\rangle$ is $20 \%$ and in $\sigma 10 \%$. Number of runs (No.) in an ensemble of 12 runs showing relatively strong spin-up of the flow.

\begin{tabular}{llllll}
\hline \hline$b$ & 1.0 & 0.95 & 0.9 & 0.8 & 0.7 \\
\hline$\langle A\rangle$ & 0.07 & 0.17 & 0.31 & 0.58 & 0.68 \\
$\langle A\rangle_{c}$ & & 0.29 & 0.52 & 0.58 & 0.68 \\
$\sigma$ & 0.002 & 0.011 & 0.022 & 0.045 & 0.073 \\
No. & 0 & 2 & 6 & 12 & 12 \\
\hline \hline
\end{tabular}

reached. All runs for $b=0.8$ show fairly rapid spin-up followed by a slow spin-down due to long-term dissipation. While the total kinetic energy is continuously dissipated, the flow maintains normalized angular momentum by assuming a more uniformlike rotation. The oscillations of the angular momentum, as observed in several runs during the spin-up process in an elliptic geometry with minor axis $b=0.8$, are related to the formation of a domain-sized tripole that interacts with the domain boundary. This phenomenon has been observed and explained earlier in the square bounded geometry [7], and we suspect that a similar mechanism is responsible for the oscillations in angular momentum signal for the flow in the elliptic geometry. The right-hand panel in Fig. 4 shows two examples of the angular momentum versus time for $b=0.9$, so for an ellipse with a smaller eccentricity. Not all the runs for this case show strong spin-up events [six runs show spin-up with an amplitude of $A \approx 0.5$ (see Table I for definition of $A$ ) and six runs show only weak spin-up, $A$ $\approx 0.2]$ and the ensemble averaged amplitude is smaller than for the $b=0.8$ case. In Fig. 5, the vorticity and stream function are given for a run showing strong spin-up and a run with weak spin-up. Note that the corresponding time series

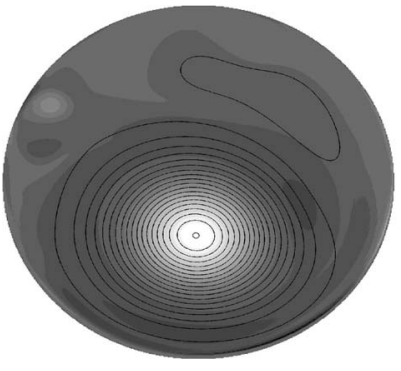

spin-up

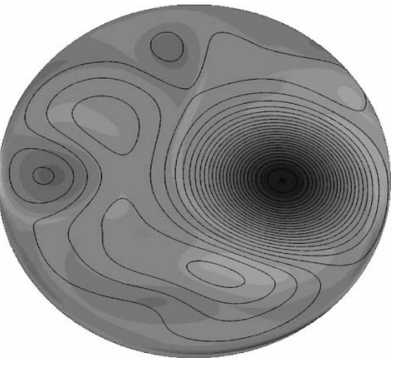

weak spin-up
FIG. 5. Vorticity and stream function of two runs with slightly different initial conditions in an elliptic geometry with minor halfaxis $b=0.9$ at $\tau=500$. The run in the left-hand panel shows a strong spin-up event (corresponding with the thin lines in Fig. 4 case $b$ $=0.9$ ); the run shown in the right-hand panel shows only weak spin-up (corresponding with the thick lines in Fig. 4 case $b=0.9$ ). White indicates positive vorticity and black negative vorticity; for both runs, the vorticity ranges from $\omega=-5$ to $\omega=+5$. Isolines represent the stream function with an increment of 0.01 . of the angular momentum is presented in the right-hand panel of Fig. 4 for the case $b=0.9$. It can be observed in Fig. 5 that a strong spin-up can be associated with the development of a strongly asymmetric dipole. On the other hand, in the case of a weak spin-up an asymmetric quadrupole configuration can be recognized inside the elliptic container. Recall that this quadrupolar structure is also found in the end state of the nonlinear regime (but far from the Stokes regime) in a circular geometry $(b=1.0)$ where spin-up is virtually absent $[3,5,8,19]$. This observation is confirmed by the present simulations for $b=1.0$ : no runs in the ensemble of 12 runs show spin-up. Spin-up becomes rare for the intermediate case $b=0.95$ (two runs with amplitude $A \approx 0.3$ in an ensemble of 12 runs).

Figure 6 shows the probability density function (pdf) of the derivative of the angular momentum $\dot{L}(t)$, which is essentially equal to the net torque on the container. It can be deduced from Fig. 6 that on average the net torque is Gaussian distributed (the histogram of the torque of a specific run can strongly deviate from Gaussian behavior, especially in the tails of the distribution). Table I presents the ensemble averaged amplitude $\langle A\rangle$ and standard deviation $\sigma$ of the derivative of the angular momentum. The standard deviation $\sigma$ of the Gaussian fit curves corresponds to the second-order moment of the ensemble averaged distribution. It appears that $\sigma$ depends linearly on the deviation of $b$ from the unit circle for $\delta=1-b \leqq 0.2$. Recall that this scaling behavior can be related to the prefactor (proportional to $\delta$ ) in the pressure contribution in Eq. (3). The standard deviation for the case $b=0.7(\delta=0.3)$ seems (considering a $10 \%$ error margin in $\sigma)$ to be larger than expected from the prefactor alone. Note that a spin-up of the flow could enhance symmetry breaking on the domain boundary and thus increase, in turn, the magnitude of the pressure contribution in Eq. (3).

The relative importance of the pressure term and the contribution of the viscous shear and normal stresses for the standard deviation $\sigma$ has been checked. It is found that the standard deviation of the viscous term in the angular momentum balance (3) is always less than 0.001 , which implies that

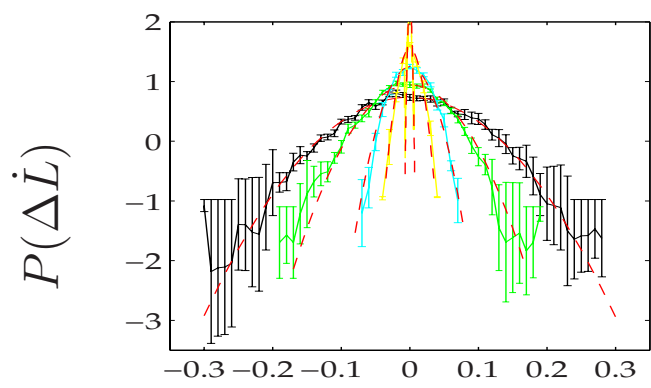

\section{$\Delta \dot{L}$}

FIG. 6. (Color online) Semilogarithmic plot of the pdf (solid lines) of $\dot{L}(t)$ for different sizes of minor half-axis $b$. The first pdf in the center corresponds with $b=1.0$; when moving outward $b$ equals $0.95,0.9,0.8$, and 0.7 , respectively. The pdf is averaged over a time interval $\tau=[5,100]$. Error bars are based on computations from an ensemble of 12 runs for each value of $b$. Gaussian fit (dashed) to the ensemble averages. 
the production of angular momentum is predominantly a result of the pressure contribution.

In order to investigate the Reynolds number dependence, a smaller number of ensemble runs is performed for $\mathrm{Re}=2$ $\times 10^{4}$ using a minor half-axis $b=0.9$. It is observed that the intensity of the spin-up process does not increase significantly. The standard deviation of $\dot{L}$ determined over the same time interval as used in Fig. 6 is $\sigma_{b=0.9}=2.5 \times 10^{-2}$, which lies within a $10 \%$ error margin of $\sigma$ computed for $\operatorname{Re}=10^{4}$. Furthermore, the frequency of strong spin-up events (four) within the ensemble (nine runs) is also similar to the Re $=10^{4}$ case.

\section{CONCLUSION}

The results presented in this paper are helpful to understand the strikingly different behavior of the angular momentum in square versus circular geometries. By using a volumepenalization technique, it is possible, starting with a circular geometry, to gradually introduce some eccentricity. It is demonstrated that a small transition from the circle results in a linear increase of the magnitude of the torque (in terms of the standard deviation), which can be related to the relative importance of the pressure contribution in the balance that guides the angular momentum production. In this respect, the observations reported on the absence of significant spin-up in a circular geometry and the associated ambivalence in the end state $[2,3,8]$ are robust, i.e., the eccentricity cannot be regarded as a bifurcation parameter in this respect. Recall that it might be conjectured that an introduction of very small eccentricity may result in a symmetry breaking of the flow and significantly enhance the magnitude of the integral over the domain boundaries in Eq. (3). Apparently, it is the prefactor in front of the pressure contribution in Eq. (3), however, that tunes the magnitude of the torque and in addition the strength of the spin-up. When moving from a circular toward a noncircular geometry, this gives a gradual transition from virtually no spin-up toward a regime where all the runs in an ensemble with slightly different initial conditions show strong and rapid spin-up events. The eccentricity above which all runs show spin-up can be denoted by the critical value of the minor axis, and the present results clearly indicate that $0.8 \leq b_{\text {crit }} \leqslant 0.9$. Between those limiting cases, thus $b_{\text {crit }} \leqslant b \leqslant 1$, there exists a critical sensitivity to the initial conditions. Some runs in the ensemble show strong spin-up, whereas the other runs in the ensemble demonstrate very weak or virtually no spin-up. Recall that the initial flow of all the ensemble runs does not contain angular momentum within machine precision accuracy. Small variations of the initial conditions are introduced by a slight displacement of the core of the Gaussian vortices on a regular lattice. It is thus surprising that these small differences in the initial conditions can result in a markedly different end state of the decay process. The number of spin-up events increases significantly for increasing eccentricities.

In previous reports, it was anticipated that the pressure contribution depends only weakly on the Reynolds number since the pressure will obviously reach finite values in the limit of infinite Reynolds numbers [7]. The analysis of the torque for the ensemble study at a significantly higher Reynolds number $\left(\mathrm{Re}=2 \times 10^{4}\right)$ supports this conjecture. In addition, the probability that the flow will demonstrate strong and rapid spin-up is not markedly affected by the Reynolds number of the flow.

It may be interesting to note that apparently the discontinuity of the domain boundaries (corners) is not essential for breaking the symmetry of the flow. By changing the eccentricity, it is possible to tune the relative importance of the pressure and viscous stresses in the angular momentum balance. The present study convincingly shows that angular momentum production is essentially due to the pressure contribution at the boundaries. As a consequence, the results of statistical mechanical studies that usually consider inviscid flow with free-slip boundaries may be more generally relevant for the quasistationary final states of viscous flow in bounded domains with no-slip sidewalls, though in a qualitative sense. A statistical mechanical prediction of the quasistationary end state of inviscid flow in an elliptic geometry with free-slip boundaries, in the spirit of Pointin and Lundgren [20] and Chavanis and Sommeria [21], would therefore be an interesting endeavor.

\section{ACKNOWLEDGMENTS}

One of the authors (G.H.K.) gratefully acknowledges financial support from the Dutch Foundation for Fundamental Research on Matter (FOM). This work was sponsored by the National Computing Facilities Foundation (NCF) for the use of supercomputer facilities with the financial support from the Netherlands Organization for Scientific Research (NWO).
[1] G. J. F. van Heijst, H. J. H. Clercx, and D. Molenaar, J. Fluid Mech. 554, 411 (2006).

[2] S. Li and D. Montgomery, Phys. Lett. A 218, 281 (1996).

[3] S. Li, D. Montgomery, and W. Jones, Theor. Comput. Fluid Dyn. 9, 167 (1997).

[4] S. Li, D. Montgomery, and W. Jones, J. Plasma Phys. 56, 615 (1996).

[5] S. R. Maassen, H. J. H. Clercx, and G. J. F. van Heijst, Europhys. Lett. 46, 339 (1999).
[6] H. J. H. Clercx, S. R. Maassen, and G. J. F. van Heijst, Phys. Rev. Lett. 80, 5129 (1998).

[7] H. J. H. Clercx, A. H. Nielsen, D. J. Torres, and E. A. Coutsias, Eur. J. Mech. B/Fluids 20, 557 (2001).

[8] K. Schneider and M. Farge, Phys. Rev. Lett. 95, 244502 (2005).

[9] D. Montgomery, Lecture Notes in Pure and Applied Mathematics (Chapman \& Hall/CRC, New York, 2006), Vol. 250, Chap. 5. 
[10] G. B. Arfken and H. J. Weber, Mathematical Methods for Physicists (Elsevier, Amsterdam, 2005).

[11] E. Arquis and J. P. Caltagirone, Numer. Math. 81, 497 (1984).

[12] G. Carbou and P. Fabrie, Adv. Differ. Equ. 8, 1453 (2003).

[13] P. Angot, C. H. Bruneau, and P. Fabrie, Numer. Math. 81, 497 (1999).

[14] K. Schneider, Comput. Fluids 34, 1223 (2005).

[15] N. K. R. Kevlahan and J.-M. Ghidaglia, Eur. J. Mech. B/Fluids 20, 333 (2001).

[16] C. H. Bruneau and H. Kellay, Phys. Rev. E 71, 046305 (2005).

[17] G. H. Keetels, U. D’Ortona, W. Kramer, H. J. H. Clercx, K.
Schneider, and G. J. F. van Heijst, J. Comput. Phys. 227, 919 (2007).

[18] G. H. Keetels, H. J. H. Clercx, and G. J. F. van Heijst, Int. J. Multiscale Comp. Eng. 6, 53 (2008).

[19] S. R. Maassen, H. J. H. Clercx, and G. J. F. van Heijst, Phys. Fluids 14, 2150 (2002).

[20] Y. B. Pointin and T. S. Lundgren, Phys. Fluids 19, 1459 (1976).

[21] P. H. Chavanis and J. Sommeria, J. Fluid Mech. 314, 267 (1996). 